\section{Abfallwirtschaft - alles ist im Fluss}

\section{Einführung zum Schwerpunkt- thema}

\section{von Ludwig Leible, ITAS}

Aufgrund der Vielschichtigkeit in der Wahrnehmung bestehender Probleme in der Abfallwirtschaft und der vielfältigen Faktoren, die ihre derzeitige Entwicklung und Dynamik bestimmen, wurde dieses Thema als Schwerpunkt des vorliegenden Heftes gewählt. Darüber hinaus werden zur Zeit in ITAS verschiedene systemanalytische Untersuchungen durchgeführt, die in diesen Themenblock einzuordnen sind.

Wesentliche Größen, die für die Abfallwirtschaft entscheidend sind, lassen sich vereinfacht in drei Bereiche einordnen, die teilweise in sehr ausgeprägter Wechselwirkung zueinander stehen. Dies sind die Bereiche

- gesellschaftliche Resonanz und Akzeptanz,

- politische, gesetzliche und wirtschaftliche Rahmenbedingungen und

- technische Entwicklungen.

Die einzelnen Beiträge zu diesem Themenschwerpunkt lassen sich relativ zwanglos in eine solche Gliederung einordnen.

Zunächst stellt Bernd Martens in seinem Beitrag "Gesellschaftliche Resonanz auf das Abfallproblem: Wahrnehmungen und Thematisierungsprozesse in einer historischen Perspektive" das Abfallproblem in einen historischen Rahmen. Er zeigt, wie sich der gesellschaftliche Umgang mit Abfallstoffen im Laufe der vergangenen zwei Jahrhunderte und insbesondere in den 90er Jahren gewandelt hat. Die Frage, welche Tendenz sich in Zukunft durchsetzen wird - Abfälle primär als ökonomisches oder als ökologisches Problem in einer über- greifenden Perspektive (Stichwort: Nachhaltigkeit) - bleibt seiner Ansicht nach offen.

Dass der Bereich Abfall und Abfallwirtschaft von einer zunehmenden Regelungsdichte durch Gesetze, Verordnungen oder Richtlinien betroffen ist, wird aus nahezu allen Beiträgen dieses Schwerpunktthemas deutlich. Dietrich Brune beleuchtet hierzu unter der Überschrift "Neuere Entwicklungen in der Abfallgesetzgebung der EU" einige bedeutende supranationale Regelungen, die in den letzten zwei Jahren in Kraft getreten sind bzw. sich noch im Gesetzgebungsverfahren befinden.

Auf nationaler Ebene gehen Bernd Bilitewski mit seinem Beitrag "Stand und Prognosen der Entsorgungssituation für Siedlungsabfälle in Deutschland" und Klaus Fricke und Thomas Turk unter der Überschrift "Stand und Perspektiven der biologischen Abfallverwertung und -behandlung in Deutschland" ebenfalls auf rechtliche Rahmenbedingungen der Abfallwirtschaft ein. Im Vordergrund ihrer Beiträge stehen jedoch neben der Beschreibung der Mengenperspektiven in der Abfallbehandlung und -verwertung insbesondere die Probleme, die aus der Umsetzung der Technischen Anleitung Siedlungsabfall (TASi) resultieren. Es wird eine Modifizierung der TASi gefordert, die auch die Deponierung von mechanisch-biologisch behandelten Abfällen erlaubt. Bei strenger Auslegung der TASi ist ab 2005 nur noch die Deponierung von thermisch behandelten Abfällen möglich.

Welche Bedeutung die Logistik und die Konditionierung von Abfällen für deren Behandlung und Verwertung hat, wird in den beiden Beiträgen von Dornbusch et al. "Logistik: Erfassung und Transport von Abfällen" und von Ulrich Loll "Konditionierung von Klärschlämmen und anderen biogenen Abfällen zur stofflichen Verwertung bzw. energetischen Nutzung" beleuchtet. Dornbusch et al. gehen insbesondere auf technische Ansätze ein, um einerseits die Erfassungsqualität von Wertstoffen zu verbessern, andererseits aber auch, um mehr Verursachergerechtigkeit bei den Abfallgebühren $\mathrm{zu}$ praktizieren und Optimierungsmöglichkeiten hinsichtlich Kostensenkung zu erschließen. Bei der Beschreibung der wichtigsten Behandlungstechniken zur Konditionierung von Abfällen geht Loll neben den verfahrenstechnischen Zusammenhängen auch auf 
deren Leistungsgrenze und die dazugehörigen gesetzlichen Anforderungen ein.

Leible et al. präsentieren in ihrem Artikel "Energetische Nutzung von biogenen Abfällen" erste Ergebnisse einer systemanalytischen Studie, die derzeit in ITAS durchgeführt wird. Nach kurzem Bezug auf aktuelle Entwicklungen in der Abfallwirtschaft und politische Zielsetzungen hinsichtlich der Nutzung erneuerbarer Energieträger werden erste Ergebnisse zum derzeitigen Aufkommen biogener Abfälle und $\mathrm{zu}$ ihrer energetischen Nutzung zur Stromgewinnung vorgestellt. Auf die technisch möglichen Potentiale wird eingegangen. Dadurch, dass exemplarische Ergebnisse aus dem Bereich der Erfassung und Konditionierung biogener Abfälle mit eingeschlossen sind, wird dem Leser - im Gegensatz zu den beiden vorangegangenen Beiträgen - eine geschlossene Kette vom Aufkommen bis zur Nutzung der Abfälle aufgezeigt.

Abschließend wird dem Forschungszentrum Karlsruhe an dieser Stelle die Gelegenheit gegeben, seine derzeitigen Forschungsaktivitäten im Themenfeld Abfall kurz vorzustellen. Arendt et al. konzentrieren sich in ihrem Beitrag "Forschungsthema Abfall - Überblick über die Arbeiten im Forschungszentrum Karlsruhe" auf den Bereich der thermischen Behandlung und energetischen Verwertung von Siedlungs-, Industrie- und landwirtschaftlichen Abfällen, ergänzt um Arbeiten zur Wiedergewinnung von Wertstoffen aus der Bau- und Metallindustrie. Diese Arbeiten haben hinsichtlich ihrer Ausrichtung vor allem anwendungs-, aber auch grundlagenorientierten Forschungscharakter.

Wie der Titel "Abfallwirtschaft - alles ist im Fluss" andeutet, ist es das Ziel dieses Schwerpunktes, dem Leser mit der Auswahl der einzelnen Beiträge einen Überblick über dieses durch eine ausgeprägte Dynamik und vielfältige Wechselwirkungen gekennzeichnete Themenfeld zu geben. $\mathrm{Ob}$ er jedoch nach der Lektüre der Einschätzung wird folgen können, dass vieles - oder gar alles - im Fluss ist, bleibt offen.

$\gg$
Gesellschaftliche Resonanz auf das Abfallproblem: Wahrnehmungen und Thematisierungsprozesse in einer historischen Perspektive

\author{
von Bernd Martens, Universität Karlsruhe
}

Es wird auf ein historisches Phasenmodell Bezug genommen, um gesellschaftliche Resonanzprozesse auf das Abfallproblem in einem größeren Kontext darzustellen. Insbesondere die Entwicklung in der Bundesrepublik Deutschland seit den 90er Jahren wird berücksichtigt, indem Umfrageergebnisse, Leitfadeninterviews und Medienanalysen herangezogen werden.

\section{Einleitung}

Die folgenden Ausführungen basieren auf meiner Habilitationsschrift "Die gesellschaftliche Resonanz auf das Abfallproblem" (Martens 1999). Dort werden auf unterschiedlichen analytischen Ebenen und empirischen Grundlagen Veränderungen im gesellschaftlichen Umgang mit Abfallstoffen nachgezeichnet. Theoretische und empirische Ansätze werden genutzt, um eine Beschreibung der gesellschaftlichen Resonanz auf das Abfallproblem, in der Bundesrepublik Deutschland in den 90er Jahren, zu geben. So werden anhand von Leitfadeninterviews mit Mitarbeitern und Leitern von Abfallwirtschaftsämtern Sichtweisen und Deutungsmuster dargestellt, die mit der Etablierung und den Wandlungen des Politikfeldes "Abfallwirtschaft" verbunden sind. In einer wissenssoziologischen Perspektive und auf der Grundlage von Befragungsdaten werden Sichtweisen kollektiver Akteure analysiert. Schließlich wird der Diskurs über das Abfallproblem mittels einer Medienanalyse aufgegriffen, bei der auf der Basis von Zeitungsartikeln zum Thema "Abfall" in der Lokal- und Regionalpresse die Darstellung der Problematik in der Öffentlichkeit eine Berücksichtigung erfährt.

Den zeitlichen Kontext der Analysen bilden Umbrüche in der Abfallwirtschaft, die seit Ende der 80er Jahre in der Bundesrepublik Deutschland in der Medienöffentlichkeit breiter diskutiert wurden. Diese Phase, die durch die 